\title{
Pharmacotherapy for Treatment and Prevention of Proliferative Diabetic Retinopathy
}

\author{
Jason Flamendorf • Howard F. Fine
}

Published online: 11 September 2014

(C) Springer Science + Business Media New York 2014

\begin{abstract}
Proliferative diabetic retinopathy poses a major public health burden with panretinal photocoagulation the only standard of care. Given the recent studies demonstrating the effectiveness of anti-vascular endothelial growth factor (VEGF) therapy for treating diabetic macular edema, pharmacotherapy may also be useful for prevention and treatment of proliferative diabetic retinopathy. Anti-VEGF therapy shows the greatest promise with its ability to delay retinopathy progression and even lead to improvements in disease severity. Furthermore, it may be useful for inducing regression of retinal and iris neovascularization. Corticosteroids, tetracyclines, and ocriplasmin are other treatments that have been considered. Additional randomized controlled trials are needed to support routine use of pharmacotherapy for specific indications in patients with proliferative diabetic retinopathy.
\end{abstract}

Keywords Proliferative diabetic retinopathy .

Pharmacotherapy · Anti-vascular endothelial growth factor (VEGF) - Triamcinolone - Tetracyclines · Ocriplasmin

\section{Introduction}

Diabetic retinopathy (DR) is the leading cause of new onset vision loss in adults 20-74 years of age in the United States

J. Flamendorf

Icahn School of Medicine at Mount Sinai, One Gustave L. Levy

Place, New York, NY 10029, USA

H. F. Fine $(\bowtie)$

Rutgers-Robert Wood Johnson Medical School,

125 Paterson St., New Brunswick, NJ 08901, USA

e-mail: hffine@yahoo.com

H. F. Fine

NJ Retina, 10 Plum Street \#600, New Brunswick, NJ 08901, USA and a leading cause worldwide [1, 2]. Among the US population 40 years and older with diabetes, $28.5 \%$ have some degree of retinopathy, and $1.5 \%$ have proliferative diabetic retinopathy (PDR) [1]. A meta-analysis showed that the global prevalence is even higher with $34.6 \%$ of individuals 20-79 years old with any DR and $6.96 \%$ with PDR [3]. Although recent advances in management have helped to lessen progression to PDR and vision-threatening levels of retinopathy $[4,5]$, the risk of retinopathy progression is still alarmingly high. Six-year follow-up of an African American cohort found that $15.0 \%$ had developed PDR over that time period, and a study in an English population reported a similar but slightly lower incidence of $11.0 \%$ over 10 years [6, 7]. Twenty-five year follow-up of type 1 diabetics from the Wisconsin Epidemiologic Study of Diabetic Retinopathy showed that $42 \%$ had progressed, although the young age at diagnosis $(<30$ years old) may inflate the progression rate relative to the overall diabetic population [8]. Nevertheless, these studies have shown that more severe retinopathy at baseline is associated with greater risk of progressing to PDR, which is particularly concerning given the significant underutilization of vision care services by individuals diagnosed with diabetes, especially African Americans [9, 10]. Furthermore, worsening disease severity is significantly associated with negative impact on health-related quality of life, higher risk of cardiovascular death and non-fatal events, and greater cognitive decline [11-13].

A number of randomized clinical trials have recently established the effectiveness of anti-vascular endothelial growth factor (VEGF) therapy, particularly ranibizumab, in the treatment of diabetic macular edema (DME) [14••, 15, $16,17]$. Once PDR develops, panretinal photocoagulation (PRP) remains the standard of care established by the Diabetic Retinopathy Study (DRS) [18]. While efficacious, PRP 
has a number of potential adverse effects, including exacerbation of macular edema, peripheral visual-field defects, and nyctalopia [19], and its administration can be difficult in the presence of vitreous hemorrhage (VH), a complication of retinal neovascularization [20, 21]. Other sight-threatening complications of PDR are tractional retinal detachment (TRD), which, along with non-clearing VH, can be managed by vitrectomy, and neovascular glaucoma [22]. There are currently no pharmacologic agents FDA approved for the treatment of PDR, but there is increasing interest in their utility considering the large public health burden PDR poses, the shortcomings and risks of current treatments, and the efficacy of anti-VEGF therapy for DME. This review will summarize the current literature on the use of pharmacotherapy for the prevention and treatment of PDR.

\section{Anti-VEGF Therapy}

\section{VEGF in Proliferative Diabetic Retinopathy}

Chronic hyperglycemia in diabetes activates multiple aberrant biochemical and cellular pathways resulting in microvascular dysfunction and retinal ischemia. In the absence of oxygenation, tissues upregulate VEGF, a potent signaling molecule that plays an integral role in angiogenesis by inducing endothelial cell proliferation, migration, and tube formation. Even before retinal neovascularization develops, increased VEGF in DR leads to degradation of endothelial cell-cell adhesions, which causes vessel leakage and macular edema [23].

Samples from human eyes have established the importance of VEGF in PDR. Significantly elevated levels of VEGF were demonstrated in vitreous and ocular-fluid samples collected at the time of intraocular surgery from patients with active PDR when compared to individuals with quiescent PDR, NPDR, or non-diabetic patients [24, 25]. Injection of $\mathrm{VEGF}_{165}$, an abundant splice variant of VEGF, into the eyes of non-human primates caused development of phenotypic features of NPDR and PDR, including pre-retinal and iris neovascularization [26-28]. Control eyes did not develop any DR-related pathology, and a dose-response relationship with VEGF was observed. Finally, murine and non-human primate models of ischemic retinopathy were used to demonstrate that VEGF-neutralizing antibodies reduced retinal and iris neovascularization [29, 30]. Taken in aggregate, these studies provide a strong basis for investigating anti-VEGF agents for PDR.

\section{Diabetic Retinopathy Progression}

As previously mentioned, ranibizumab has been extensively studied for the treatment of DME, and while changes in visual acuity were the primary outcomes of these studies, several randomized clinical trials have included DR progression as a secondary outcome. These results provide insight into the efficacy of anti-VEGF agents in preventing or delaying onset of PDR. RIDE and RISE [14••], two parallel phase III multicenter trials, randomized 759 participants with DME and study eye BCVA 20/40-20/320 into one of three treatment arms: sham injections, $0.3-\mathrm{mg}$ intravitreal ranibizumab, and $0.5-\mathrm{mg}$ intravitreal ranibizumab. Injections were given monthly, and starting at month 3 , all patients were evaluated monthly for as-needed macular laser based on protocol-specified criteria. One outcome for evaluating DR progression was the ETDRS severity scale level that was assigned based on grading of color fundus photos. At the 2-year primary endpoint, 15.0 and $11.5 \%$ of participants in the sham groups of RISE and RIDE had progressed to PDR, while 1.6-5.6\% in the ranibizumab groups of both trials progressed $(p=0.0001-0.0206$ for comparisons with the sham groups).

A separate analysis by Ip et al. [31••] found that the ranibizumab groups were significantly less likely to worsen and significantly more likely to improve by $\geq 2$ and $\geq 3$ steps on the severity scale. In addition, the median severity level decreased in both ranibizumab groups while it remained at "moderately severe NPDR" in the sham groups, and the percent of patients with mild NPDR increased more substantially in the ranibizumab groups compared to the sham group. Other outcomes indicating progression to PDR were also considered, including receiving PRP, occurrence of $\mathrm{VH}$, performance of vitrectomy for PDR-related sequelae, and development of iris or retinal neovascularization-related complications. Combining these other outcomes resulted in $33.8 \%$ of sham-treated eyes versus $11.2-11.5 \%$ of ranibizumab-treated eyes developing PDR ( $p<0.0001)$. Shamtreated patients were more likely to receive PRP, develop $\mathrm{VH}$, or undergo vitrectomy.

RIDE and RISE were only randomized for the first 2 years since patients in the sham groups were allowed to crossover to receive $0.5 \mathrm{mg}$ ranibizumab. Nevertheless, results at the end of year 3 showed that in both ranibizumab groups, 37.8-40.9\% of eyes improved by $\geq 2$ steps and $11.3-15.4 \%$ by $\geq 3$ steps [15]. This is compared with 23.4 and $24.3 \%$ in RIDE and RISE, respectively, who had an improvement of $\geq 2$ steps and 2.6 and $4.0 \%$ for $\geq 3$ step improvements among the sham groups. Finally, the sham crossover group demonstrated improvements in DR severity after receiving ranibizumab.

The DRCR.net has also investigated the efficacy of ranibizumab for preventing retinopathy progression. A study of 854 eyes of 691 participants with DME compared $0.5 \mathrm{mg}$ intravitreal ranibizumab plus prompt (3-10 days after injection) or deferred ( $\geq 24$ weeks after injection) focal/grid photocoagulation and $4 \mathrm{mg}$ intravitreal 
triamcinolone plus prompt macular laser with sham injection plus prompt laser [16]. Injections were given every 4 weeks for the first 12 weeks and then were administered PRN as often as every 4 weeks based on a specified protocol for retreatment. At 1 year, ranibizumab-treated eyes were less likely to demonstrate progression of retinopathy compared to the sham group based on color fundus photo grading. Furthermore, the ranibizumab group was less likely to have a $\mathrm{VH}$ or receive PRP compared to the sham group.

Follow-up of the initial study population was continued for 3 years, and an exploratory analysis was performed to evaluate the risk of worsening of DR [32•]. The criteria for progression to PDR were expanded to include: worsening by $\geq 2$ steps on the ETDRS severity scale, receiving PRP, development of $\mathrm{VH}$, or vitrectomy for PDR. There were 538 of 792 eyes $(67.9 \%)$ that did not have PDR at baseline, and among these eyes, the ranibizumab groups had significantly lower cumulative probabilities of progressing to PDR at the end of years 1, 2, and 3. Similar results were observed in the cohort with baseline PDR, and statistically significant hazard ratios of 0.43 and 0.42 were calculated for ranibizumab plus prompt or deferred laser, respectively, compared to the sham group. A major limitation to this study is that fundus photographs at 2 and 3 years were available for only 6 and $50 \%$ of the eyes, respectively, due to a change in the protocol related to the beneficial effect of ranibizumab on DME. As such, the proportion of individuals at these time points with worsening of DR are likely underestimated.

\section{Adjunct to PRP}

PRP is hypothesized to exert its effect by improving oxygenation of the inner retina, subsequently decreasing VEGF production and inducing regression or stabilization of neovascularization [33]. Anti-VEGF agents have also been reported to induce regression of retinal neovascularization in PDR. Uncontrolled case series have demonstrated that bevacizumab could induce complete regression of neovascularization, as assessed by ophthalmoscopy and/ or fluorescein angiography, in $61.4-100 \%$ of participants. Regression could occur as early as 1 week and as late as 1 month post-injection, with cases of recurrence 2 weeks to 3 months after initial treatment [34-40]. Filho et al. [41] conducted a randomized controlled trial comparing PRP to PRP with ranibizumab given as a single $0.5 \mathrm{mg}$ injection after the first of two laser sessions; retreatment with ranibizumab in the combined group and additional PRP in the laser only group were given at 16 and 32 weeks after baseline. At 48 weeks, total area of fluorescein leakage, the primary outcome, was reduced in both groups compared to baseline, but the reduction was significantly greater with
PRP plus ranibizumab $(p=0.0291)$. This group also demonstrated better visual acuity outcomes and a reduction in central subfield macular thickness (CSMT) compared to the PRP only group, which showed worsening of visual acuity and an increase in CSMT. A similar study with bevacizumab demonstrated a significant reduction in neovascularization but no differences in BCVA compared to PRP alone [42].

Another potential role for anti-VEGF as an adjunct to PRP is for the synergistic effect it may have on reducing exacerbations of macular edema. One of the largest studies, conducted by the DRCR.net, compared ranibizumab and triamcinolone with sham injections in patients with severe NPDR or PDR and existing DME who received focal/grid photocoagulation at baseline and PRP at least 2 weeks later [43•]. At the 14-week primary endpoint, the ranibizumab group $(n=113)$ had a mean visual acuity change of +1 letter compared to -4 letters for the sham group $(p<0.0001)$, and the mean CSMT decreased by significantly more in eyes treated with ranibizumab $(-39 \mu \mathrm{m}$ vs. $-5 \mu \mathrm{m}$ for sham, $p=0.01$ ). A retrospective review by Mason et al. [44] found similar results for bevacizumab in 60 eyes with severe PDR receiving two sessions of PRP \pm bevacizumab, showing a significantly higher BCVA and significantly lower foveal thickness for the combination group at 24 weeks follow-up ( $p \leq 0.0001$ for both comparisons).

\section{Regression of Rubeosis}

In addition to retinal neovascularization, rubeosis in the context of PDR has been noted to respond to anti-VEGF therapy. A case series of 28 eyes noted that 20 (71.4\%) had rubeosis that responded to bevacizumab, while smaller studies reported higher response rates. Regression could be observed as early as 1 day post-treatment, although some patients required 2 or 3 monthly injections before any effect was seen. Studies that had long enough follow-up reported recurrence in some patients, but 4 patients followed for 12 months did not have any recurrence after receiving 3 monthly ranibizumab injections. Finally, the effect on IOP was variable, with some case series finding that it was well controlled during short-term follow-up and others requiring medical and/or surgical interventions [4549].

\section{Vitreous Hemorrhage}

Anti-VEGF treatments have also been investigated for their use in resolving $\mathrm{VH}$, which can prevent administration of PRP and may necessitate vitrectomy. Uncontrolled case series have reported positive results, with hemorrhage partially clearing as early as 1 week after treatment and remaining clear for 1-9 months post-treatment, with some 
patients requiring multiple injections [34-37]. A larger study of 40 eyes with persistent $\mathrm{VH}$ was compared to a historical control group after receiving one or two intravitreal ranibizumab injections; the treatment group demonstrated a significantly shorter vitreous clear-up time and a significantly lower rate of vitrectomy [50]. The DRCR.net recently carried out a double-masked, randomized controlled trial comparing $0.5 \mathrm{mg}$ ranibizumab to saline since it has been hypothesized that clearance of $\mathrm{VH}$ may be due to the intravitreal injection itself and not antiVEGF activity [51]. At 16 week follow-up, the cumulative probabilities of vitrectomy were not significantly different between the two groups (ranibizumab $12 \%$ vs. saline $17 \%, p=0.37$ ), although the ranibizumab group was more likely to have completed PRP (44 vs. $31 \%$, $p=0.05)$ and to experience a greater increase in mean visual acuity (22 letters vs. 16 letters, $p=0.04$ ). At 1 year follow-up, both the cumulative probability of vitrectomy and the mean change in visual acuity did not demonstrate significant differences between the two groups [52]. One limit to this study is that the rates of vitrectomy were lower than expected for both groups, so it may have been underpowered to detect a treatment difference.

\section{Adjunct to Vitrectomy}

Anti-VEGF therapy prior to vitrectomy has been investigated for its potential in improving surgical outcomes and reducing postoperative $\mathrm{VH}$. A study by di Lauro et al. [53] randomized 72 eyes of 68 patients to receive a sham injection or $1.25 \mathrm{mg}$ of bevacizumab 7 or 20 days prior to vitrectomy for VH or TRD. Intraoperative management was improved for bevacizumab administered at either time point before surgery compared to the sham group in terms of intraoperative bleeding ( $8.3 \%$ and 12.5 vs. $79.1 \%, p<0.0001$ for both comparisons), use of endodiathermy ( $8.3 \%$ and 12.5 vs. $54.1 \%, p<0.0001$ for both comparisons), and operating time (65 min and 69 vs. $84 \mathrm{~min}, p=0.025$ and 0.031 ). Zhang et al. [54] found similar results by conducting a metaanalysis of 8 randomized controlled trials, including the di Lauro et al. study, comparing vitrectomy with and without preoperative bevacizumab. The authors found a shorter overall surgical time (mean difference $=-26.89 \mathrm{~min}$, $p<0.0001$ ), smaller number of endodiathermy applications (mean difference $=-3.46, p=0.02$ ), less intraoperative bleeding risk $(\mathrm{OR}=0.10, p=0.003)$, and lower risk of recurrent VH within the first month $(\mathrm{OR}=0.35$, $p<0.0001$ ) for preoperative treatment with bevacizumab. No difference in the VH recurrence was observed after the first month. In contrast to this meta-analysis, Ahn et al. [55] found no significant decrease in risk of recurrent $\mathrm{VH}$ within 4 weeks of vitrectomy among 107 eyes randomized to preoperative or post-vitrectomy bevacizumab compared with vitrectomy alone ( $22.2 \%$ and 10.8 vs. $32.4 \%, p=0.087)$. No significant differences in recurrence rates were observed more than 4 weeks after vitrectomy, either, with the postvitrectomy bevacizumab group actually experiencing a higher risk of recurrence.

\section{Safety}

One of the main safety concerns that has arisen in the context of anti-VEGF use for PDR is the potential for development or progression of TRD, possibly due to rapid neovascular involution with accelerated fibrosis. A retrospective review of 211 patients who had received intravitreal bevacizumab for PDR refractory to PRP found that 11 (5.2\%) developed or had progression of TRD 3-31 days post-treatment, although the authors acknowledged this could have happened by natural history [56]. Van Geest et al. [57] measured VEGF and connective tissue growth factor (CTGF) in vitreous samples from patients with PDR, some of whom received bevacizumab preoperatively; they found that VEGF was negatively correlated and CTGF positively correlated with fibrosis and that patients receiving bevacizumab had more fibrosis $(p<0.0001)$. There is also a concern about administering anti-VEGF therapy to diabetic patients who are already at increased risk of cardiovascular events and mortality. Large clinical trials have found that ocular adverse events (AEs) are similar in ranibizumab and sham-treated groups, including incidence of TRD, although event rates are typically low making it difficult to rule out a small difference in risk $[14 \bullet \bullet, 15,16,17]$. The 36 -month data from RISE and RIDE also revealed a potentially increased risk of non-fatal cardiovascular events and overall incidence of death in patients receiving the $0.5 \mathrm{mg}$ dose, but this was not found with the FDA-approved dose of $0.3 \mathrm{mg}$.

Based on current available evidence, it appears that antiVEGF therapy may have a role in preventing and treating PDR (Table 1). Treated eyes in large clinical trials of DME showed a decrease in the risk of retinopathy progression and even demonstrated improvements on the ETDRS severity scale. These studies, however, were not designed with the primary objective of determining who would benefit most from anti-VEGF injections as prevention for PDR, and any conclusions about disease progression and regression are limited to a patient population with DME at baseline. Once PDR has developed, anti-VEGF therapy can induce regression of retinal and iris neovascularization, although the evidence is largely based on case series. A search of ClinicalTrials.gov \{performed August 2014\} showed several trials, at various stages of completion, that are evaluating the effects of different combinations of ranibizumab and prompt or deferred PRP on regression of neovascularization or reduction of macular edema (NCT01280929, NCT01941329, NCT01594281, NCT01489189). Pending 
Table 1 Anti-VEGF therapy in proliferative diabetic retinopathy

\begin{tabular}{|c|c|}
\hline Indication & Current evidence \\
\hline $\begin{array}{l}\text { Retinopathy } \\
\text { progression }\end{array}$ & $\begin{array}{l}\text { Significantly less likely to progress to PDR, mor } \\
\text { likely to improve } \geq 2 \text { or } \geq 3 \text { steps on the } \\
\text { ETDRS severity scale, and less likely to } \\
\text { experience complications of PDR }\end{array}$ \\
\hline Adjunct to PRP & $\begin{array}{l}\text { Limited evidence to suggest improved regression } \\
\text { of neovascularization and greater improvements } \\
\text { in BCVA compared to PRP alone. Better } \\
\text { BCVA and CSMT outcomes when used prior to } \\
\text { PRP }\end{array}$ \\
\hline $\begin{array}{l}\text { Regression of } \\
\text { rubeosis }\end{array}$ & $\begin{array}{l}\text { Case series demonstrating regression of rubeosis } \\
\text { with variable recurrence and effects on IOP }\end{array}$ \\
\hline $\begin{array}{l}\text { Vitreous } \\
\text { hemorrhage }\end{array}$ & $\begin{array}{l}\text { No benefit over intravitreal saline in a } \\
\text { randomized clinical trial }\end{array}$ \\
\hline $\begin{array}{l}\text { Adjunct to } \\
\text { vitrectomy }\end{array}$ & $\begin{array}{l}\text { Improved intraoperative outcomes but evidence } \\
\text { equivocal regarding reduction in } \mathrm{VH} \text { recurrence }\end{array}$ \\
\hline
\end{tabular}

the results of these studies, anti-VEGF as an adjunct to PRP, or perhaps a first-line treatment, may gradually become a standard of care for high-risk PDR, although the potential increased risk of TRD from inducing rapid regression must be studied further. Finally, current evidence does not support a role for anti-VEGF therapy in treating $\mathrm{VH}$ or for reducing recurrence of $\mathrm{VH}$ when used preoperatively for vitrectomy, although it may improve intraoperative outcomes.

\section{Anti-Inflammatory Therapy}

\section{Rationale}

Inflammation has a central role in the pathogenesis of DR. Rat models of diabetes have demonstrated that leukostasis, attraction and adhesion of leukocytes to the vascular wall, is temporally and spatially related to capillary nonperfusion in the retina, and ICAM-1, the molecule mediating leukocyte adherence to the endothelium, is upregulated in diabetic humans and animals [58, 59]. Numerous proinflammatory enzymes (i.e., iNOS, COX-2), transcription factors (i.e., NF- $\kappa \beta$ ), cytokines (i.e., IL- $1 \beta$, TNF $\alpha$ ), and lipid mediators (i.e., prostaglandins, leukotrienes) are also upregulated. This leads to retinal capillary degeneration and blood-retinal barrier breakdown, which results in edema. VEGF is also proinflammatory, and its expression is increased by cytokine release, suggesting that inhibiting VEGF works on the inflammatory component of DR while anti-inflammatory agents would further reduce VEGF [60].

\section{Corticosteroids}

The DRCR.net has performed exploratory analyses of clinical trials focusing on DME to investigate the effect, if any, that triamcinolone has on DR progression. One study in which patients were randomized to 1 or $4 \mathrm{mg}$ of intravitreal triamcinolone or focal/grid photocoagulation calculated the cumulative probability of progressing to PDR based on reading center grading, receiving PRP, developing $\mathrm{VH}$, or worsening by $\geq 2$ steps on the ETDRS scale [61]. At 3 years of follow-up, the probabilities were 35, 30, and $37 \%$ for the $1 \mathrm{mg}, 4 \mathrm{mg}$, and laser groups, respectively, with the only significant difference between the $4 \mathrm{mg}$ and laser groups $(p=0.02)$. Another study, previously discussed, examining ranibizumab plus prompt or deferred macular laser also compared $4 \mathrm{mg}$ of triamcinolone plus prompt laser [16]. An exploratory analysis showed that among eyes without PDR at baseline, the cumulative probability of worsening retinopathy was significantly lower at 1 year for the triamcinolone group compared to sham plus prompt laser, but this difference was not maintained at 2 and 3 years, with the triamcinolone group ultimately having a higher rate of progression (37 vs. $23 \%$ ) [32•]. The subgroup analysis among eyes with PDR at baseline demonstrated a significantly lower probability of progression for triamcinolone at all followup visits.

Corticosteroids have also been investigated as an adjunct to PRP to reduce macular edema in eyes with pre-existing DME. The DRCR.net found that during short-term (14 weeks) follow-up, eyes receiving triamcinolone plus macular laser had a significant improvement in mean visual acuity and a significant decrease in mean central subfield thickness compared to laser alone [43•]. Mirshahi et al. [62] did not find any difference in BCVA or central macular thickness from baseline to 6-month follow-up comparing eyes receiving PRP and macular laser \pm triamcinolone pretreatment 1 week earlier. Finally, one study examined posterior sub-Tenon triamcinolone for patients with PDR undergoing vitrectomy for retinal detachment and found no differences in reattachment rate, visual acuity, or postoperative complications compared to a non-randomized control group at 6 months follow-up [63]. Overall, the results regarding corticosteroids are mixed, and given the significant ocular AEs of cataract formation and IOP elevation [16, $43,61]$, as well as the efficacy of anti-VEGF therapy for the same indications, routine steroid use does not seem warranted at this time.

\section{Tetracyclines}

Microglia are the resident immune cells of the retina and become activated in DR, contributing to the inflammatory process [64-66]. Tetracyclines, best known as antibiotics, have demonstrated anti-inflammatory and neuroprotective effects in animal models and cell culture, including inhibition of microglial morphologic changes at low doses [67, 
68]. Scott et al. [69] carried out a small proof-of-concept trial in which 30 patients with severe NPDR or non-highrisk PDR were randomized to 24 months of $50 \mathrm{mg}$ doxycycline or placebo once daily. They found no significant differences between the two groups with respect to change in visual acuity, change in CSMT, or disease progression. The drug was well-tolerated. An uncontrolled Phase I/II study of 5 patients treated with minocycline for DME showed modest improvements in BCVA and CSMT in almost all eyes over 6 months without the need for macular laser and/or anti-VEGF therapy [70]. These results are encouraging and warrant further investigation given the potential for an oral medication to reduce the number of intravitreal injections required.

\section{Pharmacologic Vitreolysis}

Retinal neovascularization requires a scaffold to grow onto the posterior surface of the vitreous [71]. Eyes that have a complete posterior vitreous detachment (PVD) are less likely to develop neovascular disease and experience PDR progression [72]. The period when a PVD is evolving, however, is a time of increased risk for the development of complications from PDR. If vitreous liquefaction and proteolysis releasing the posterior vitreous cortex from the internal limiting membrane do not occur in concert, excessive traction on the retina can lead to VH, TRD, and/or DME [73]. Ocriplasmin is a recombinant serine protease FDA approved for one-time use in vitreomacular traction syndrome. The most relevant outcome measure from clinical trials is the rate of total PVD since partial PVD in patients with PDR could still facilitate neovascularization. In the Phase II trials, total PVD was induced in 24 and $31 \%$ of eyes receiving the FDAapproved dose of $125 \mu \mathrm{g}$ compared with 8 and $10 \%$ in the sham groups; the Phase III trial showed $13.4 \%$ of drug-treated eyes and $3.7 \%$ of sham-treated eyes had total PVD at day 28 [74-76]. Few studies have investigated pharmacologic vitreolysis in DR, and most have used autologous plasmin enzyme, which is larger and less stable than ocriplasmin [77]. Two case series found 38 and $41.7 \%$ of eyes had total PVD after one injection, $50 \%$ with PDR showed significant neovascularization regression, and success of PVD was significantly correlated with a thinner, less reflective posterior vitreous face $[78,79]$. Pharmacologic vitreolysis may be more effective in diabetic eyes than in non-diabetic eyes with vitreomacular traction, but larger double-masked, placebocontrolled trials are required to obtain more definitive evidence of efficacy. Further consideration could be warranted in patients with complications of PDR who are not good candidates for vitrectomy.
Table 2 Non-anti-VEGF therapies in proliferative diabetic retinopathy

\begin{tabular}{|c|c|}
\hline Treatment & Current evidence \\
\hline Corticosteroids & $\begin{array}{l}\text { Equivocal evidence for preventing retinopathy } \\
\text { progression and for improving outcomes when used } \\
\text { prior to PRP. No evidence for use as adjunct to } \\
\text { vitrectomy. Significant ocular AEs include cataract } \\
\text { formation and IOP elevation }\end{array}$ \\
\hline Tetracyclines & $\begin{array}{l}\text { Uncontrolled trial for DME showed modest } \\
\text { improvements in BCVA and CSMT. Current evidence } \\
\text { does not support use in PDR }\end{array}$ \\
\hline Ocriplasmin & $\begin{array}{l}\text { Case series suggest some efficacy for inducing total } \\
\text { PVD and regression of neovascularization but no } \\
\text { randomized clinical trials to date specifically } \\
\text { addressing use in PDR }\end{array}$ \\
\hline
\end{tabular}

Future Directions

While targeting VEGF has shown great efficacy in treating DME and promise of preventing PDR or treating its complications, there is increasing interest in other potential targets for drug design. A study of VEGF-independent cytokines in vitreous of PDR patients found that 16 cytokines other than VEGF were significantly associated with the presence of PDR [80]. Topical ketorolac given to patients undergoing vitrectomy for complications of PDR showed an ability to significantly reduce vitreous levels of IL-8 and PDGF-AA, cytokines that may contribute to PDR [81]. The PI3K/Akt/mTOR pathway has been implicated in angiogenesis, and sirolimus has been well-tolerated in small studies for DME, suggesting that it could also be investigated in PDR [82, 83]. Finally, blockade of the renin-angiotensin system with enalapril and losartan in patients with type 1 diabetes produced significant reductions in retinopathy progression by $\geq 2$ steps on the ETDRS scale [84].

\section{Conclusion}

FDA approval of pharmacologic agents for prevention or treatment of PDR seems a distant goal, but results of completed and continuing studies may soon advocate for the use of pharmacotherapy for select indications. Anti-VEGF medications appear to be the most promising treatments, but the pathogenesis of DR is complex and involves a plethora of inflammatory mediators, signaling molecules, and cell types (Table 2). Exploring other targets for drug design may expand the PDR armamentarium to maximize visual outcomes and provide greater variety in the methods of administration.

Disclosure Jason Flamendorf and Howard F. Fine both have no reported conflict of interests.

Human and Animal Rights and Informed Consent This article does not contain any studies with human or animal subjects performed by any of the authors. 


\section{References}

Recently published papers of particular interest have been highlighted as:

- Of importance

-. Of major importance

1. Zhang X, Saadine JB, Chou CF, et al. Prevalence of diabetic retinopathy in the United States, 2005-2008. JAMA. 2010;304:649-56.

2. Resnikoff S, Pascolini D, Eyta'ale D, et al. Global data on vision impairment in the year 2002. Bull World Health Organ. 2004;82:844-51.

3. Yau JW, Rogers SL, Kawasaki R, et al. Global prevalence and major risk factors of diabetic retinopathy. Diabetes Care. 2012;35:556-64.

4. LeCaire TJ, Palta M, Klein R, et al. Assessing progress in retinopathy outcomes in type 1 diabetes: comparing findings from the Wisconsin Diabetes Registry Study and the Wisconsin Epidemiologic Study of Diabetic Retinopathy. Diabetes Care. 2013;36:631-7.

5. Wong TY, Mwamburi M, Klein R, et al. Rates of progression in diabetic retinopathy during different time periods: a systematic review and meta-analysis. Diabetes Care. 2009;32:2307-13.

6. Roy MS, Affouf M. Six-year progression of retinopathy and associated risk factors in African American patients with type 1 diabetes mellitus: the New Jersey 725. Arch Ophthalmol. 2006; 124:1297-306.

7. Jones CD, Greenwood RH, Misra A, et al. Incidence and progression of diabetic retinopathy during 17 years of a population-based screening program in England. Diabetes Care. 2012;35:592-6.

8. Klein R, Knudtson MD, Lee KE, et al. The Wisconsin Epidemiologic Study of Diabetic Retinopathy: XXII the 25-year progression of retinopathy in persons with type 1 diabetes. Ophthalmology. 2008;115:1859-68.

9. Bressler NM, Varma R, Doan QV, et al. Underuse of the health care system by persons with diabetes mellitus and diabetic macular edema in the United States. JAMA Ophthalmol. 2014;132:168-73.

10. Maclennan PA, McGwin G Jr, Heckemeyer C, et al. Eye care use among a high-risk diabetic population seen in a public hospital's clinics. JAMA Ophthalmol. 2014;132:162-7.

11. Mazhar K, Varma R, Choudhury F, et al. Severity of diabetic retinopathy and health-related quality of life: the Los Angeles Latino Eye Study. Ophthalmology. 2011;118:649-55.

12. Gerstein HC, Ambrosius WT, Danis R, et al. Diabetic retinopathy, its progression, and incident cardiovascular events in the ACCORD trial. Diabetes Care. 2013;36:1266-71.

13. Ding J, Strachan MW, Reynolds RM, et al. Diabetic retinopathy and cognitive decline in older people with type 2 diabetes: the Edinburgh Type 2 Diabetes Study. Diabetes. 2010;59:2883-9.

14. •• Nguyen QD, Brown DM, Marcus DM, et al. Ranibizumab for diabetic macular edema: results from 2 phase III randomized trials: RISE and RIDE. Ophthalmology. 2012;119:789-801. This study demonstrates that ranibizumab decreases the risk of progressing to proliferative diabetic retinopathy based on results from two large randomized controlled trials following patients for 2 years.

15. Brown DM, Nguyen QD, Marcus DM, et al. Long-term outcomes of ranibizumab therapy for diabetic macular edema: the 36-month results from two phase III trials: RISE and RIDE. Ophthalmology. 2013;120:2013-22.

16. Diabetic Retinopathy Clinical Research Network. Randomized trial evaluating ranibizumab plus prompt or deferred laser or triamcinolone plus prompt laser for diabetic macular edema. Ophthalmology. 2010;117:1064-77.

17. Mitchell P, Bandello F, Schmidt-Erfurth U, et al. The RESTORE study: ranibizumab monotherapy or combined with laser versus laser monotherapy for diabetic macular edema. Ophthalmology. 2011;118:615-25.

18. Diabetic Retinopathy Study Research Group. Photocoagulation treatment of proliferative diabetic retinopathy: clinical application of Diabetic Retinopathy Study Research Group. Arch Ophthalmol. 1979;97:654-5.

19. Bressler NM, Beck RW, Ferris FL 3rd. Panretinal photocoagulation for proliferative diabetic retinopathy. $N$ Engl J Med. 2011;365:1520-6.

20. Flynn HW, Chew EY, Simons BD, et al. Pars plana vitrectomy in the Early Treatment Diabetic Retinopathy Study: ETDRS Report No. 17. Ophthalmology. 1992;99:1351-7.

21. Adamis AP, Aiello LP, D'Amato RA. Angiogenesis and ophthalmic disease. Angiogenesis. 1999;3:9-14.

22. Ho T, Smiddy WE, Flynn HW Jr. Vitrectomy in the management of diabetic eye disease. Surv Ophthalmol. 1992;37:190-202.

23. Penn JS, Madan A, Caldwell RB, et al. Vascular endothelial growth factor in eye disease. Prog Retin Eye Res. 2008;27:331-71.

24. Adamis AP, Miller JW, Bernal MT, et al. Increased vascular endothelial growth factor levels in the vitreous of eyes with proliferative diabetic retinopathy. Am J Ophthalmol. 1994;118:445-50.

25. Aiello LP, Avery RL, Arrigg PG, et al. Vascular endothelial growth factor in ocular fluid of patients with diabetic retinopathy and other retinal disorders. N Engl J Med. 1994;331:1480-7.

26. Toletino MJ, Miller JW, Gragoudas ES, et al. Vascular endothelial growth factor is sufficient to produce iris neovascularization and neovascular glaucoma in a nonhuman primate. Arch Ophthalmol. 1996;114:964-70.

27. Toletino MJ, Miller JW, Gragoudas ES, et al. Intravitreous injections of vascular endothelial growth factor produce retinal ischemia and microangiopathy in an adult primate. Ophthalmology. 1996;103:1820-8.

28. Toletino MJ, McLeod DS, Taomoto M, et al. Pathologic features of vascular endothelial growth factor-induced retinopathy in nonhuman primate. Am J Ophthalmol. 2002;133:373-85.

29. Adamis AP, Shima DT, Toletino MJ, et al. Inhibition of vascular endothelial growth factor prevents retinal ischemia-associated iris neovascularization in a nonhuman primate. Arch Ophthalmol. 1996;114:66-71.

30. Aiello LP, Pierce EA, Foley ED, et al. Suppression of retinal neovascularization in vivo by inhibition of vascular endothelial growth factor (VEGF) using soluble VEGF-receptor chimeric proteins. Proc Natl Acad Sci USA. 1995;92:10457-61.

31. • Ip MS, Domalpally A, Hopkins JJ, et al. Long-term effects of ranibizumab on diabetic retinopathy severity and progression. Arch Ophthalmol. 2012;130:1145-1152. This analysis of the RISE and RIDE results showed that ranibizumab not only delays progression of but also improves diabetic retinopathy severity. The paper also analyzed progression to proliferative diabetic retinopathy using extended criteria.

32. - Bressler SB, Qin H, Melia M, et al. Exploratory analysis of the effect of intravitreal ranibizumab or triamcinolone on worsening of diabetic retinopathy in a randomized clinical trial. JAMA Ophthalmol. 2013;131:1033-1040. This paper investigates how ranibizumab or triamcinolone affects progression to proliferative diabetic retinopathy when combined with prompt or deferred macular laser for DME at baseline.

33. Aiello LP. Angiogenic pathways in diabetic retinopathy. N Engl J Med. 2005;353:839-41.

34. Spaide RF, Fisher YL. Intravitreal bevacizumab (Avastin) treatment of proliferative diabetic retinopathy complicated by vitreous hemorrhage. Retina. 2006;26:275-8. 
35. Moradian S, Ahmadieh H, Malihi M, et al. Intravitreal bevacizumab in active progressive proliferative diabetic retinopathy. Graefes Arch Clin Exp Ophthalmol. 2008;246:1699-705.

36. El-Batarny AM. Intravitreal bevacizumab treatment for retinal neovascularization and vitreous hemorrhage in proliferative diabetic retinopathy. Clin Ophthalmol. 2007;1:149-55.

37. Minnella AM, Savastano CM, Ziccardi L, et al. Intravitreal bevacizumab (Avastin) in proliferative diabetic retinopathy. Acta Ophthalmol. 2008;86:683-7.

38. Mason JO 3rd, Nixon PA, White MF. Intravitreal injection of bevacizumab (Avastin) as adjunctive treatment of proliferative diabetic retinopathy. Am J Ophthalmol. 2006;142:685-8.

39. Avery RL, Pearlman J, Pieramici DJ, et al. Intravitreal bevacizumab (Avastin) in the treatment of proliferative diabetic retinopathy. Ophthalmology. 2006;113:1695 e1-15.

40. Arevalo JF, Wu L, Sanchez JG, et al. Intravitreal bevacizumab (Avastin) for proliferative diabetic retinopathy: 6-months followup. Eye (Lond.). 2009;23:117-23.

41. Filho JAR, Messias A, Almeida FPP, et al. Panretinal photocoagulation (PRP) versus PRP plus intravitreal ranibizumab for high-risk proliferative diabetic retinopathy. Acta Ophthalmol. 2011;89:e567-72.

42. Tonello M, Costa RA, Almeida FPP, et al. Panretinal photocoagulation versus PRP plus intravitreal bevacizumab for high-risk proliferative diabetic retinopathy (IBeHi study). Acta Ophtahlmol. 2007;86:385-9.

43. - Diabetic Retinopathy Clinical Research Network. Randomized trial evaluating short-term effects of intravitreal ranibizumab or triamcinolone acetonide on macular edema follow focal/grid laser for diabetic macular edema in eyes also receiving panretinal photocoagulation. Retina. 2011;31:1009-27. This randomized controlled trial demonstrates that patients receiving ranibizumab or triamcinolone as an adjunct to PRP and focal/grid laser had better visual acuity and less macular edema.

44. Mason JO 3rd, Yunker JJ, Vail R, et al. Intravitreal bevacizumab (Avastin) prevention of panretinal photocoagulation-induced complications in patients with severe proliferative diabetic retinopathy. Retina. 2008;28:1319-24.

45. Oshima Y, Sakaguchi H, Gomi F, et al. Regression of iris neovascularization after intravitreal injection of bevacizumab in patients with proliferative diabetic retinopathy. Am J Ophthalmol. 2006;142:155-8.

46. Grisanti S, Biester S, Peters S, et al. Intracameral bevacizumab for iris rubeosis. Am J Ophthalmol. 2006;142:158-60.

47. Brouzas D, Charakidas A, Moschos M, et al. Bevacizumab (Avastin) for the management of anterior chamber neovascularization and neovascular glaucoma. Clin Ophthalmol. 2009;3:685-8.

48. Jiang Y, Liang X, Li X, et al. Analysis of the clinical efficacy of intravitreal bevacizumab in the treatment of iris neovascularization caused by proliferative diabetic retinopathy. Acta Ophthalmol. 2009;87:736-40.

49. Tu Y, Fay C, Guo S, et al. Ranibizumab in patients with dense cataract and proliferative diabetic retinopathy with rubeosis. Oman J Ophthalmol. 2012;5:161-5.

50. Huang YH, Yeh PT, Chen MS, et al. Intravitreal bevacizumab and panretinal photocoagulation for proliferative diabetic retinopathy associated with vitreous hemorrhage. Retina. 2009;29:1134-40.

51. Diabetic Retinopathy Clinical Research Network. Randomized clinical trial evaluating intravtireal ranibizumab or saline for vitreous hemorrhage from proliferative diabetic retinopathy. JAMA Ophthalmol. 2013;131:283-93.

52. Bhavsar AR, Torres K, Glassman AR, et al. Evaluation of results 1 year following short-term use of ranibizumab for vitreous hemorrhage due to proliferative diabetic retinopathy. JAMA Ophthalmol. 2014;132:889-90.
53. di Lauro R, De Ruggiero P, di Lauro R, et al. Intravitreal bevacizumab for surgical treatment of severe proliferative diabetic retinopathy. Graefes Arch Clin Exp Ophthalmol. 2010;248: 785-91.

54. Zhang ZH, Liu HY, Hernandez-Da Mota SE, et al. Vitrectomy with or without preoperative intravitreal bevacizumab for proliferative diabetic retinopathy: a meta-analysis of randomized controlled trials. Am J Ophthalmol. 2013; 156: 106-115.e2.

55. Ahn J, Woo SJ, Chung H, et al. The effect of adjunctive intravitreal bevacizumab for preventing postvitrectomy hemorrhage in proliferative diabetic retinopathy. Ophthalmology. 2011;118: 2218-26.

56. Arevalo JF, Maia M, Flynn HW Jr, et al. Tractional retinal detachment following intravitreal bevacizumab (Avastin) in patients with severe proliferative diabetic retinopathy. $\mathrm{Br} \mathrm{J}$ Ophthalmol. 2008;92:213-6.

57. Van Geest RJ, Lesnik-Oberstein SY, Tan HS, et al. A shift in the balance of vascular endothelial growth factor and connective tissue growth factor by bevacizumab causes the angiofibrotic switch in proliferative diabetic retinopathy. $\mathrm{Br} \mathrm{J}$ Ophthalmol. 2012;96:587-90.

58. Miyamoto K, Khosrof S, Bursell SE, et al. Prevention of leukostasis and vascular leakage in streptozotocin-induced diabetic retinopathy via intercellular adhesion molecule-1 inhibition. Proc Natl Acad Sci USA. 1999;96:10836-41.

59. Kern TS. Contributions of inflammatory processes to the development of the early stages of diabetic retinopathy. Exp Diabetes Res. 2007;2007:95103.

60. Tang J, Kern TS. Inflammation in diabetic retinopathy. Prog Retin Eye Res. 2011;30:343-58.

61. Bressler NM, Edwards AR, Beck RW, et al. Exploratory analysis of diabetic retinopathy progression through 3 years in a randomized clinical trial comparing intravitreal triamcinolone with focal/grid photocoagulation. Arch Ophthalmol. 2009;127:1566-71.

62. Mirshahi A, Shenazandi H, Lashay A, et al. Intravitreal triamcinolone as an adjunct to standard laser therapy in coexisting high-risk proliferative diabetic retinopathy and clinically significant macular edema. Retina. 2010;30:254-9.

63. Lee Y, Kang S, Park YH. Posterior subtenon triamcinolone acetonide in gas-filled eyes as an adjunctive treatment for complicated proliferative diabetic retinopathy. Korean J Ophthalmol. 2013;27:28-33.

64. Rungger-Brändle E, Dosso AA, Leuenberger PM. Glial reactivity, an early feature of diabetic retinopathy. Invest Ophthalmol Vis Sci. 2000;41:1971-80.

65. Zeng XX, Ng YK, Ling EA. Neuronal and microglial response in the retina of streptozotocin-induced diabetic rats. Vis Neurosci. 2000;17:463-71.

66. Zeng HY, Green WR, Tso MO. Microglial activation in human diabetic retinopathy. Arch Ophthalmol. 2008;126:227-32.

67. Krady JK, Basu A, Allen CM, et al. Minocycline reduces proinflammatory cytokine expression, microglial activation, and caspase-3 activation in a rodent model of diabetic retinopathy. Diabetes. 2005;54:1559-65.

68. Wang AL, Yu AC, Lau LT, et al. Minocycline inhibits LPS-induced retinal microglia activation. Neurochem Int. 2005;47:152-8.

69. Scott IU, Jackson GR, Quillen DA, et al. Effect of doxycycline versus placebo on retinal function and diabetic retinopathy progression in patients with severe nonproliferative or non-high-risk proliferative diabetic retinopathy: a randomized clinical trial. JAMA Ophthalmol. 2014;132:535-43.

70. Cukras CA, Petrou P, Chew EY, et al. Oral minocycline for the treatment of diabetic macular edema (DME): results of a phase I/II clinical study. Invest Ophthalmol Vis Sci. 2012;53:3865-74. 
71. Blankenship GW, Machemer R. Long-term diabetic vitrectomy results: report of 10 year follow-up. Ophthalmology. 1985;92:503-6.

72. de Smet M, Gad El Kareem A, Zwinderman AH. The vitreous, the retinal interface in ocular health and disease. Ophthalmologica. 2013;230:165-78.

73. de Smet M, Castilla M. Ocriplasmin for diabetic retinopathy. Expert Opin Biol Ther. 2013;13:1741-7.

74. Stalmans P, Delaey C, de Smet MD, et al. Intravitreal injection of microplasmin for treatment of vitreomacular adhesion: results of a prospective, randomized, sham-controlled phase II trial (the MIVI-IIT trial). Retina. 2010;30:1122-7.

75. Benz MS, Packo KH, Gonzalez V, et al. A placebo-controlled trial of microplasmin intravitreous injection to facilitate posterior vitreous detachment before vitrectomy. Ophthalmology. 2010;117:791-7.

76. Stalmans P, Benz MS, Gandorfer A, et al. Enzymatic vitreolysis with ocriplasmin for vitreomacular traction and macular holes. N Engl J Med. 2012;367:606-15.

77. Tsui I, Pan CK, Rahimy E, et al. Ocriplasmin for vitreoretinal diseases. J Biomed Biotechnol. 2012;2012:1-6.

78. Elbendary AM, Elwan MM, Azzam HA, et al. Predictability of vitreous detachment following intravitreal plasmin injection in diabetic macular edema associated with vitreomacular traction. Curr Eye Res. 2011;36:534-9.

79. Diaz-Llopis M, Udaondo P, Millán JM, et al. Enzymatic vitrectomy for diabetic retinopathy and diabetic macular edema. World J Diabetes. 2013;4:319-23.

80. Bromberg-White JL, Glazer L, Downer R, et al. Identification of VEGF-independent cytokines in proliferative diabetic retinopathy vitreous. Invest Ophthalmol Vis Sci. 2013;54:6472-80.

81. Schoenberger SD, Kim SJ, Shah R, et al. Reduction of interleukin 8 and platelet-derived growth factor levels by topical ketorolac, $0.45 \%$, in patients with diabetic retinopathy. JAMA Ophthalmol. 2014;132:32-7.

82. Krishnadev N, Forooghian F, Cukras C, et al. Subconjunctival sirolimus in the treatment of diabetic macular edema. Graefes Arch Clin Exp Ophthalmol. 2011;249:1627-33.

83. Dugel PU, Blumenkranz MS, Haller JA, et al. A randomized, dose-escalation study of subconjunctival and intravitreal injections of sirolimus in patients with diabetic macular edema. Ophthalmology. 2012;119:124-31.

84. Mauer M, Zinman B, Gardiner R, et al. Renal and retinal effects of enalapril and losartan in type 1 diabetes. $\mathrm{N}$ Engl $\mathrm{J}$ Med. 2009;361:40-51. 